\title{
The Impact of Dynamic Retrieval Emissivity on the Microwave Surface-sensitive Channels over Land
}

\author{
De Xing ${ }^{123}$ \\ Academy of Ocean Science and Engineering, National University of Defense Technology, College of \\ Computer, National University of Defense Technology \\ Changsha, 410073, China \\ E-mail: xd wonyeicloud.com
}

\section{Qunbo Huang ${ }^{\text {a }}$ Weimin Zhang, Bainian Liu, Xiang Xing, Tengling Luo}

Academy of Ocean Science and Engineering, National University of Defense Technology, College of Computer, National University of Defense Technology

Changsha, 410073, China

E-mail: ${ }^{a}$ hqb090163.com

In addition to the traditionally complex land surface emissivity models, it is possible to direct real-time retrieve emissivity over land surface in a large-scaled temporal and spatial region from satellite microwave observations. We investigated the performance of the real-time dynamic retrieval emissivity against the monthly averaged emissivity atlas. As indicated by the results, the dynamic retrieval algorithm can obviously reduce the first guess departure of low-level channels which are sensitive to the surface and the simulated background brightness temperatures with real-time dynamic emissivity against the observations have a better agreement over the land surface and higher correlation. Therefore, the dynamic retrieval emissivity algorithm has the potential to improve the effect of satellite data assimilation.

CENet2017

22-23 July 2017

Shanghai China

\section{${ }^{1}$ Speaker}

${ }^{2}$ The study was supported by the Natural Science Foundation of China (Grant No.:

41375113).

${ }^{3}$ Correspongding Author

(C) Copyright owned by the author(s) under the terms of the Creative Commons 


\section{Introduction}

According to the detection frequency, the sensors boarding satellite at present can be mainly divided into three kinds: the visible light, the infrared and the microwave instruments. It is well known that the visible light and infrared instruments cannot provide much information in cloud and precipitation because of the limited short wavelength. However, the microwave instruments are able of penetrating through cloud and precipitation, which is beneficial to obtain the detailed atmospheric low-level state under all-sky conditions. Nevertheless, how to assimilate the satellite microwave data effectively in numerical weather forecast is still a challenging project in the world.

Mcnally pointed out that the bias correction, the emissivity modification and the cloud detection were important in the satellite data assimilation[1]. When using the microwave remote sensing to detect the atmosphere, some lower detection channels will receive electromagnetic signal contributed by surface. Consequently, it is necessary to separate the contribution part between the surface and atmosphere for the assimilation of such channels.

Currently, the majority satellite data sensitive to the surface are not operational in the world's major numerical water forecast centers. The main difficulty lies in the fact that surface emissivity exerts greater influence on the low-level channels observation and the surface emissivity is very uncertain.

The premise assimilation of low-level channels is to determine the emissivity of complicated surface[2][3]. Many scholars both at home and abroad have carried out significant researches on the retrieval of microwave surface emissivity and put forward a series of methods mostly based on physical algorithms and then in combination with the statistical analysis of the atmospheric sounding profiles. It is difficult to fully calculate various types of global surface emissivity by a single emissivity estimation model because the model is often established under limited regional surface conditions and not applicable to the large-scale application.

Another effective method is to estimate the real-time surface emissivity directly from the satellite radiance observations. The advantage of this method is that satellite can observe the earth with multi-bands and cross-sensors to get global surface information with less affected factors. At present, several surface emissivity retrieval methods based on passive microwave remote sensing are set as follows: the empirical statistical methods [4], the radiation transmission equation [5], the exponential analysis[6], the neural network[7] and the onedimensional variational methods [8].

The retrieved surface emissivity from the satellite observations is essentially the "average emissivity" in the field of view (FOV), which is important for the radiative transfer model to simulate the brightness temperature accurately. There are two major mainstream radiative transfer models in the world, namely RTTOV[9] and CRTM[10]. The former is mainly operationally applied to the institutions such as the French Meteorological Bureau, the British Meteorological Bureau and the ECMWF. The sea surface microwave emissivity model FASTEM is perfect and has already been updated to Version FASTEM6. The CRTM is principally engaged in the US NCEP. The satellite retrieval mechanism of surface emissivity involves many factors, including spatial surface type, frequency, polarization and zenith angle of observation, etc..

In this paper, based on the dynamic retrieval emissivity algorithm over the land surface, we've investigated the simulation effect of brightness temperature of low-level microwave 
channels. Section 2 describes the algorithm process of dynamically retrieving emissivity over land surface. Section 3 gives the experiment settings and analyzes the statistics features of the numerical experiment results. Finally, conclusions and plans for future work are presented in Section 5.

\section{Dynamic Retrieval Emissivity Algorithm}

The basic idea of estimating the surface emissivity by satellite microwave observation is to distinguish the individual radiative contribution between the atmosphere and surface with the assistance of atmospheric temperature, water vapor profile, surface temperature and other auxiliary data. Assuming non-scattering plane parallel atmosphere and on the basis of the approximate conditions of Rayleigh-Jeans, at the given satellite zenith angle and spectral frequency, the atmospheric radiative transfer equation is as follows[11] :

$$
\begin{gathered}
T_{b}(v, \theta)=T_{s} \varepsilon(v, \theta) \Gamma+(1-\varepsilon(v, \theta)) \Gamma T_{a}^{\downarrow}(v, \theta)+T_{a}^{\uparrow}(v, \theta)+(1-\varepsilon(v, \theta)) T_{c} \Gamma^{2} \\
\Gamma=\exp \left(\frac{-\tau(0, H)}{\cos \theta}\right)
\end{gathered}
$$

where $T_{b}$ refers to the brightness temperature observed by the satellite, $\varepsilon(v, \theta)$ refers to the surface emissivity, $T_{s}$ and $T_{c}$ correspond to the surface temperature and cosmic background brightness temperature. $T_{a}^{\downarrow}(v, \theta)$ and $T_{a}^{\uparrow}(v, \theta)$ are the atmospheric downward and upward radiative contribution respectively. $\Gamma$ refers to the transmittance from the surface to the top of the atmosphere, which is a function of satellite zenith angle $\theta$ and atmospheric opacity $\tau(0, H) . H$ refers to the height of the atmosphere top. In the past, it was difficult to describe the upward and downward radiative contribution because of limited performance of the fast radiative transfer model. However, with the development of science and technology, we're now capable of using advanced radiative transfer model to solve Equ. (2.1).

From Equ. (2.1), we can retrieve the surface emissivity:

$$
\varepsilon(v, \theta)=\frac{T_{b}(v, \theta)-T_{a}^{\uparrow}(v, \theta)-T_{a}^{\downarrow}(v, \theta) \Gamma-T_{c} \Gamma^{2}}{\left(T_{a}^{\downarrow}(v, \theta)-T_{s}-T_{c} \Gamma\right) \Gamma}
$$

The dynamic retrieval emissivity method highlights few required surface parameters and only demands the surface temperature; however, this method works perfectly only in case of clear-sky, and the inversion accuracy depends on the atmospheric background information (such as surface temperature, atmospheric temperature, humidity profile, atmospheric upward and downward radiative calculation).Fig. 1 show the flowchart of generating the emissivity atlas and real-time dynamic emissivity. A monthly mean emissivity atlas CNRM is used, as generated from the AMSU window channel observations, and the atmospheric upward and downward radiative contributions are calculated by using the fast radiative transmission model RTTOV11 based on the short-term forecast atmospheric profiles [11]. Although it has been confirmed that atlas is able to describe the spatial and temporal variation of emissivity in a certain extent, the atlas is just a climate average of emissivity in a long time series, which cannot obviously describe more detail of some complicated land surface duly. Therefore, this paper focuses on the improved effect of dynamic real-time retrieval emissivity to the atlas. 


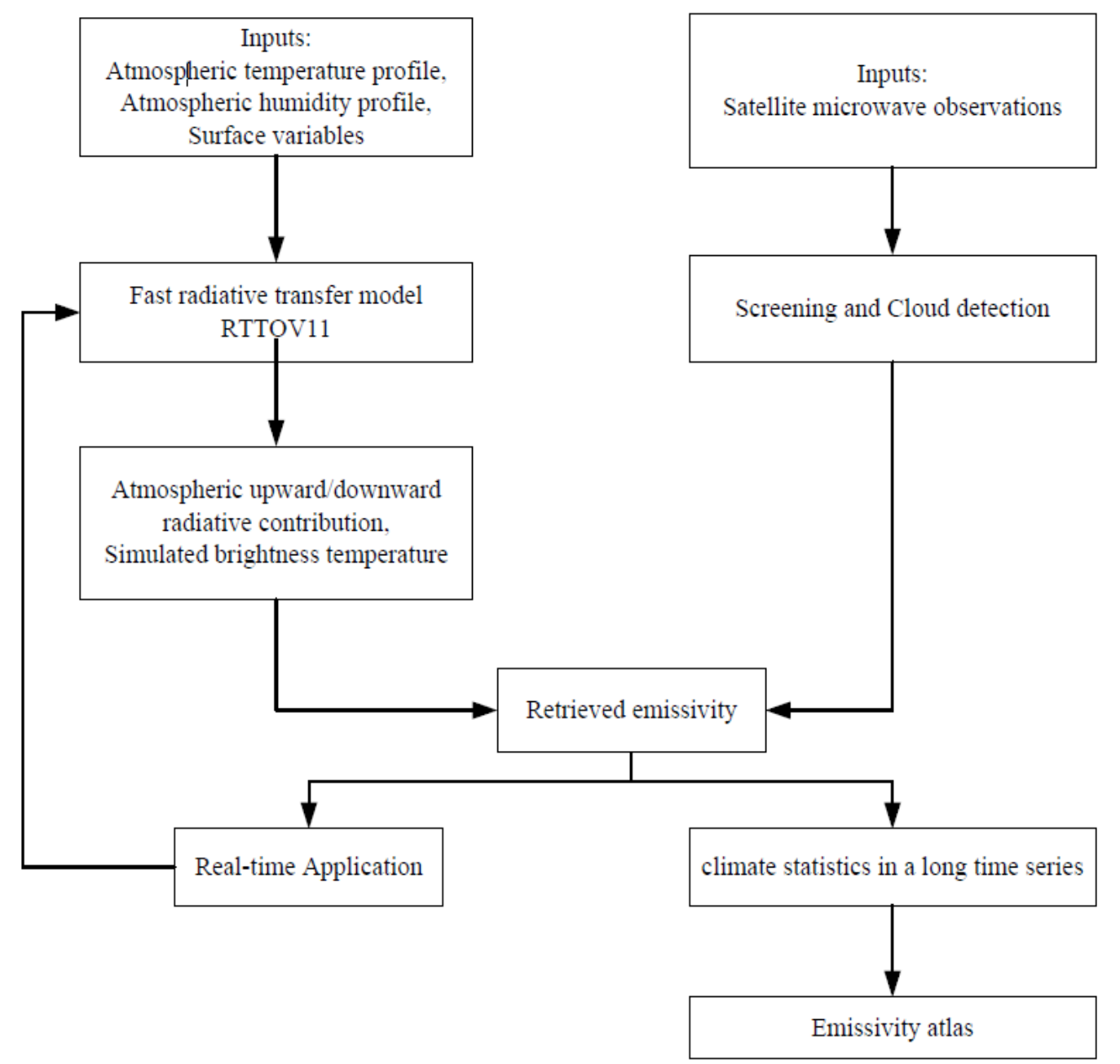

Figure 1: Flowchart of Land Surface Emissivity Retrieval from Satellite Microwave Observations

\section{Numerical Experiment}

\subsection{Experiment Setting}

The AMSU-A contained 15 channels, where Channels 1, 2, 3 and 15 are the window channels. It has been proved that the land surface emissivity of Channels 4-14 can be approximated by the retrieved emissivity of Channel 3 of the same frequency band [12]. Fig. 2 shows the weight functions of AMSU-A channels. Each curve represents the sensitivity of a specific channel to the different levels of atmosphere. It can be clearly seen from the figure that the peak energy contribution levels of the window Channels 1-3 and 15 originate from the surface and the peak energy contribution levels of Channels 4-14 are the heights of the surface to $60 \mathrm{~km}$, respectively. These curves are calculated by using the standard US atmospheric profile. [8]

According to the currently operational assimilation status on AMSU-A in the world, we simply identify the channels of AMSU-A below Channel 5 with the low-level channels which we need to study in this paper.

The observation area is about [15S, 45N] and [90E, 150E], located in Northwest Pacific and Southern China. The observation time is 2014072300 UTC. The background profile is 
generated from the short weather forecast WRF model, and finally 9976 grid points over land surface are selected from the total 24364 grid points covering all types of surface (land, sea, seaice and so on) at that time.

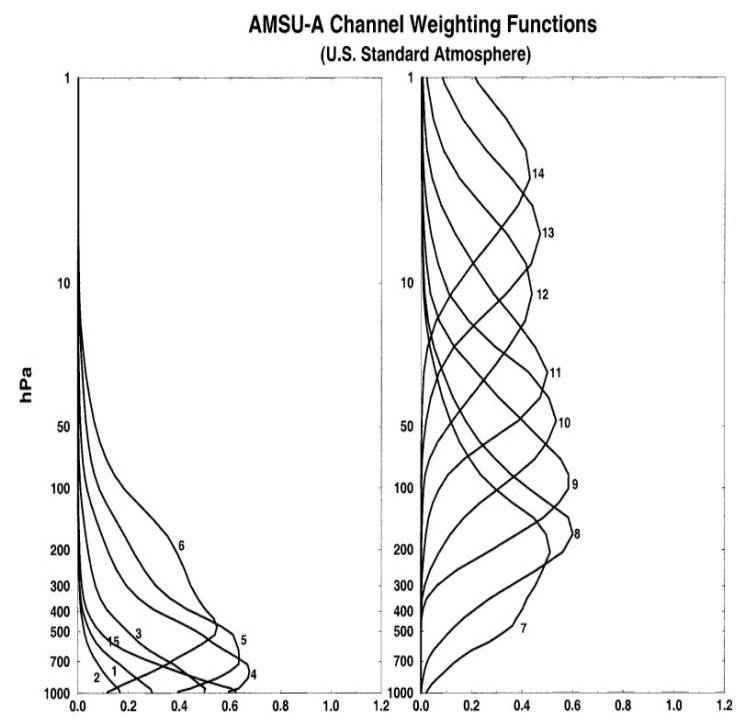

Figure 2: AMSU-A Weight Functions of Channels

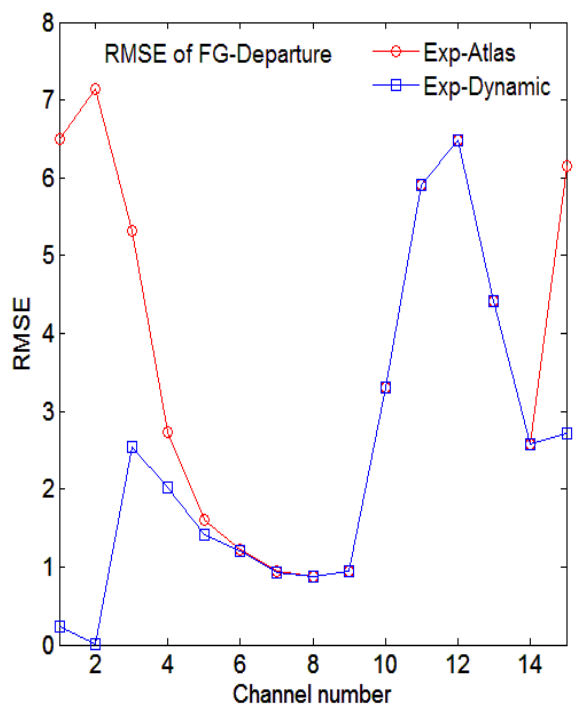

Figure 3: The RMSE of FG-Departure VS 15 AMSU-A Channels 1-15 Overland Surface.

Exp-Atlas (red line), Exp-Dynamic (blue line)

\subsection{Statistical Results}

In the field of data assimilation, the atmospheric profile produced by short-term forecast is often used as the background profile, so the departure between the real observation field and the background field is often called as the first guess departure (FG-departure, Observation minus Background).

We conduct two groups of experiment to simulate the brightness temperature with the emissivity atlas or with the dynamic emissivity, named as Exp-Atlas and Exp-Dynamic respectively.

Fig. 3 shows the root-mean-square error (RMSE) of FG-departure at each channel. It is clear that a significant improvement is obtained for the surface-sensitive channels (Channels 1, $2,3,4,5$ and 15) primarily because the primary radiative contributions of these channels originate from the surface. In the nature of things, the RMSE become smaller with more accurate surface emissivity. Fig. 4 describes the histograms of the FG-departures for Channels 1, 2, 3, 4 and 15. The results indicate that Exp-Dynamic shows better statistics, the FGdeparture values of each channel is significantly reduced (especially in the window Channel 1 and 2) in comparison with the simulated background brightness temperature in Exp-Atlas. In addition, the distribution FG-departures in Exp-Dynamic (blue dash line) come closer to the Gaussian type with more data are gathered near the zero line (black solid line). The real-time dynamic retrieval emissivity can effectively reduce the bias in priori information of the observation for the surface-sensitive channels. 

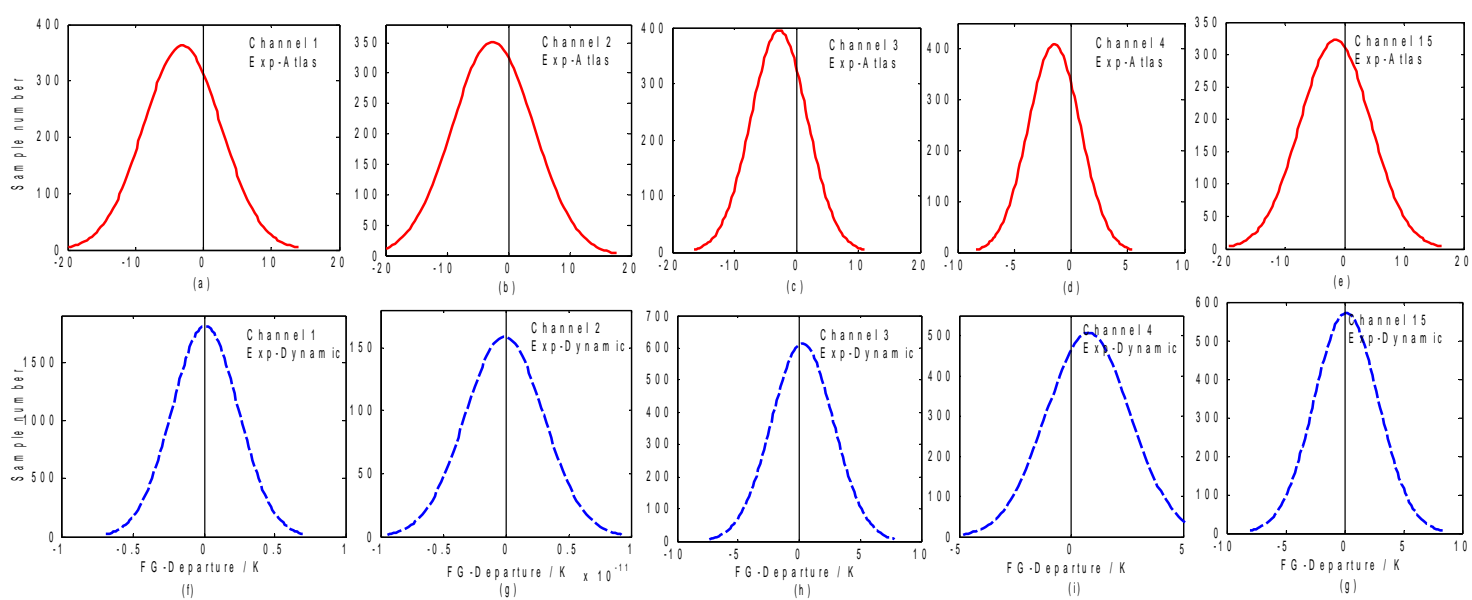

Figure 4 : Histograms of the FG-Departures for Surface Sensitive Channels 1, 2, 3, 4 and 15. Exp-Atlas (Red Solid Line) and Exp-Dyn (Blue Dash Line)

Scatter plots of simulated background brightness temperature against observation in ExpAtlas (red) and simulated background brightness temperature against observation in ExpDynamic (blue) are shown in Fig. 5. The corresponding correlation coefficients are given in Table 1. It is noticed that a better agreement on Exp-Dynamic than that of Exp-Atlas with a great increase of correlation. The distribution of points is more dispersed at $50.3 \mathrm{GHz}$ (Channel 3) than the first two channels because of the increasing atmospheric scatter effect with higher frequencies.

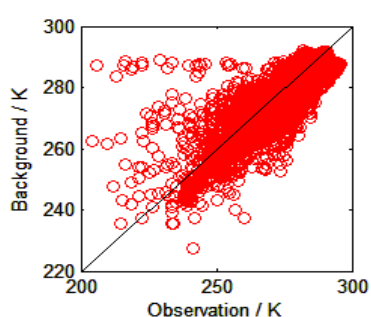

(a)

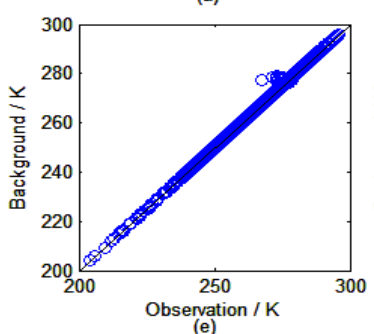

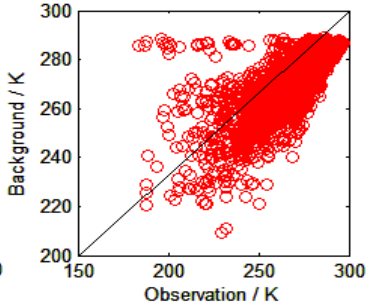

(b)

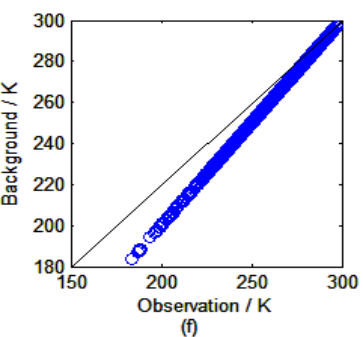

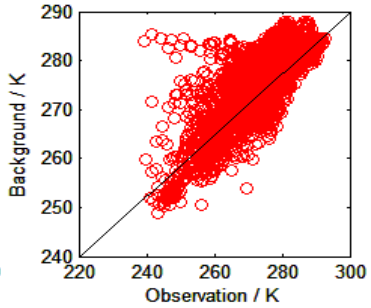

(c)

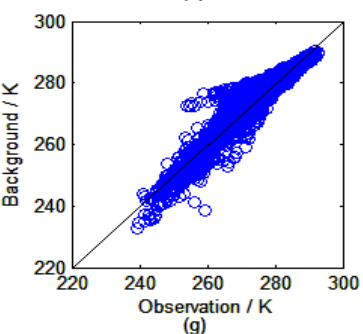

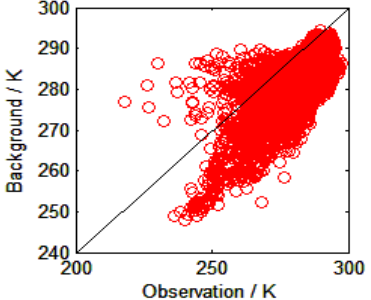

(d)

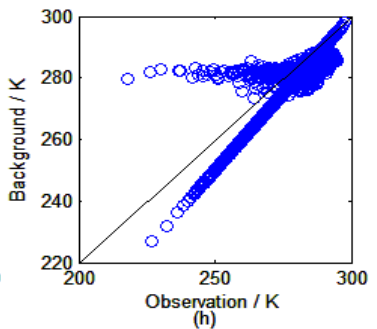

Figure 5: Scatter plots of simulated background brightness temperature in Exp-Atlas against observation (red) and simulated background brightness temperature against observation in ExpDynamic (blue)

\begin{tabular}{|c|c|c|c|c|}
\hline Correlation Coefficient & Channel 1 & Channel 2 & Channel 3 & Channel 15 \\
\hline Exp-Atlas & 0.77 & 0.73 & 0.77 & 0.71 \\
\hline Exp-Dynamic & 0.99 & 0.99 & 0.95 & 0.93 \\
\hline
\end{tabular}

Table 1: The correlation coefficients of simulated background brightness temperature against observation in Exp-Atlas and simulated background brightness temperature against observation in Exp-Dynamic Concl

\section{Conclusion}


We're expecting to obtain more accurate simulated background brightness temperature overland surface, however, it is necessary to obtain more accurate land surface emissivity at first. The results of this study suggest that the dynamic real-time updating emissivity can improve the statistics of the first guess departures at each channel for AMSU-A, therefore, better quality of the first guess brightness temperature estimations is beneficial to further improve the ability of microwave satellite data assimilation and may allow more observations to be assimilated overland surface. Many other factors (such as zenith angle and diurnal variation etc.) will affect the estimation of land surface emissivity, and it's our next research direction to retrieve the global emissivity accurately.

\section{References}

[1] A. P. Mcnally. The direct assimilation of cloud affected satellite infrared radiances in the ECMWF $4 D$-Var [J]. Quarterly Journal of the Royal Meteorological Society. 135(642): 1214-1229(2009)

[2] N. C. Grody. Surface identification using satellite microwave radiometers[J]. IEEE Transactions on Geoscience \& Remote Sensing. 26(6): 850-859(1988)

[3] F. Weng, B. Yan, N. C. Grody. A microwave land emissivity mode[J]. Journal of Geophysical Research Atmospheres. 106(D17): 20115-20124(2001)

[4] G. W. Felde, J. D. Pickle. Retrieval of 91 and $150 \mathrm{GHz}$ earth surface emissivity[J]. Journal of Geophysical Research Atmospheres. 100(D10): 20855-20866(1995)

[5] C. Prigent, W. B. Rossow, E. Matthews. Microwave land surface emissivities estimated from SSM/I observations[J]. Journal of Geophysical Research Atmospheres. 1022(D18): 21867-21890(1997)

[6] J. C. Morland, D. I. F. Grimes, T. J. Hewison. Satellite observations of the microwaveemissivity of a semi-arid land surface[J]. Remote Sensing of Environment. 77(2): 149-164(2001)

[7] F. Aires, C.Prigent, W. B. Rossow, M. Rothstein. A new neural network approach including first guess for retrieval of atmospheric water vapour, cloud liquid water path, surface temperature, and emissivities over land from satellite microwave observations[J]. Journal of Geophysical Research Atmospheres. 106(D14): 14887-14907(2001)

[8] J. R. Eyre, G. A. Kelly, A. P. Mcnally, E. Andersson, A. Persson. Assimilation of TOVS radiance information through one-dimensional variational analysis $[\mathrm{J}]$. Quarterly Journal of the Royal Meteorological Society. 119(514): 1427-1463(1993)

[9] R. Saunders, M. Matricardi, P. Brunel. An improved fast radiative transfer model for assimilation of satellite radiance observations [J]. Quarterly Journal of the Royal Meteorological Society. 125(556): 1407-1425(1999)

[10] Q. Liu, S. Boukabara. Community Radiative Transfer Model (CRTM) applications in supporting the Suomi National Polar-orbiting Partnership (SNPP) mission validation and verification [J]. Remote Sensing of Environment. 140(1): 744-754(2014)

[11] F. Karbou, C. Prigent, L. Eymard, J. R. Pardo. Microwave land emissivity calculations using AMSU measurements[J]. IEEE Transactions on Geoscience \& Remote Sensing. 43(5): 948-959(2005)

[12] F. Karbou, E. Gerard, F. Rabier. Microwave land emissivity and skin temperature for AMSU-A and$B$ assimilation over land $[\mathrm{J}]$. Quarterly Journal of the Royal Meteorological Society. 132(620): 23332355(2006) 